\title{
Black body radiation as a function of frequency and wavelength: an experimentally oriented approach
}

(Radiação de corpo negro como função da frequência e comprimento de onda: uma abordagem orientada experimentalmente)

\author{
Ademir L. Xavier Jr. ${ }^{\mathbb{1}}$ and Sergio Celaschi \\ Centro de Pesquisas Avançadas Wernher von Braun, Campinas, SP, Brasil \\ Recebido em 3/8/2011; Aceito em 8/8/2011; Publicado em 20/4/2012
}

\begin{abstract}
It is well known that the peak of black body radiation depends on the adopted description, whether as a function of frequency or wavelength. This paper is a pedagogical exposition of how to write and estimate Planck's distribution spectrum on two distinct spectrograph scales. We show that the emitted power of the Black Body maximum is unique and does not coincide with the peak measured whether in frequency or wavelength. We then make a generalization with other distribution functions in order to further clarify the apparent issue.
\end{abstract}

Keywords: Planck's law, Wien's law, black body, electromagnetic radiation.

Sabe-se que o pico de radiação de corpo negro depende da descrição adotada, a saber, se em frequência ou em comprimento de onda. Este artigo traz uma exposição pedagógica sobre como escrever e estimar o espectro da distribuição de Planck em duas escalas espectrográficas distintas. Mostramos que a potência emitida do máximo de corpo negro é única e não coincide com o pico medido, seja em frequência ou comprimento de onda. Então, fazemos uma generalização com outras funções de distribuição com o objetivo de clarificar ainda mais a questão. Palavras-chave: lei de Planck, lei de Wien, corpo negro, radiação eletromagnética.

\section{Introduction}

Every student learning the principles of Quantum Mechanics and following a historical approach is naturally impressed by Max Planck pioneering work [प]. He faced with heroism unsurmonable problems at his time to explain the Black Body (BB) radiation properties [- [0]. He successfully managed to derive a law and solve one of the greatest anomalies that paved pre-quantum physics in the beginning of the 20th century.

A dedicated student might be impressed to know that $\mathrm{BB}$ distribution law gives rise to two well distinct maxima if the $\mathrm{BB}$ spectrum is written in terms of frequency or wavelength intervals. Many books in Statistical Physics [ [ [ [3] ] prefer the frequency form. This is not surprising since Planck's distribution law is obtained from Bose-Eistein statistics of electromagnetic oscillator modes. Almost none of these books present any values at all for the resulting temperature displacement of the maximum or Wien's displacement law. An exception is Landsberg [प]]. On p. 214 (section 13.3), after deducing the frequency law, the wavelength form is obtained. However, historical accounts of the matter show that the spectrum was originally measured on a wavelength scale using a spectrograph [山్ , [6] ]. Most of

\footnotetext{
${ }^{1}$ E-mail: xavier@vonbraunlabs.com.br.

the time, derivations come from exclusively mathematical reasoning (Section 2), with scarce or no reference to the experimental setup required to register the $\mathrm{BB}$ spectrum.

It is well known that grating laws give rise to a multitude of BB maxima [ $[\mathbf{T}]$ ]. The subject has also served to motivate speculations on the causes of human vision sensitivity function [ष]. The subject is further motivated by the dependence of the BB temperature with the maximum of the spectrum. In other words, the position of this maximum is a direct measurement of the BB temperature which has several applications from material science to astrophysics or even cosmology [ㅁ] . If the peak changes position with the representation, which one should be chosen as a reference of the BB temperature? However, spectral measurements dependent strongly on experimental details that are many times absent in reference works. This paper intends to fill this gap following an experimental reasoning (that is, we numerically estimate the BB spectrum as seen by different experimental arragements, while no real experimental measurement was attempt). The approach describes the energy distribution of BB photons on spectrographic scales where wavelength are registered. In fact, there seems to exist no experimental 
linear scale for wavelength or frequency on which the BB spectrum maxima would coincide with the theoretical distribution. Indeed, we will show here that at the spectrograph there is no 'ambiguity' in the maximum peak position. In Section 2 we briefly present the problem as it is often stated without any reference to the experimental problem. In Section 3 we discuss some experimental arrangements that are employed to obtain the BB spectrum in wavelength. In Section 4 we generalize our results and make a simple but elucidating application that we hope can further clarify the problem. Finally, Section 5 presents some conclusions.

\section{BB spectrum}

Planck's distribution function [ $[$ ] gives the intensity of the emitted radiation (emitted power per unit area), per unit of a physical quantity interval (frequency, wavelength or wavenumber), from a BB at absolute temperature $T$. It takes two forms in frequency $(\nu)$ and wavelength $(\lambda)$, respectively

$$
\begin{gathered}
I_{\nu}(\nu, T)=\left(\frac{2 h \nu^{3}}{c^{2}}\right) \frac{1}{e^{h \nu / k T}-1}, \\
I_{\lambda}(\lambda, T)=\left(\frac{2 h c^{2}}{\lambda^{5}}\right) \frac{1}{e^{h c / \lambda k T}-1} .
\end{gathered}
$$

where $h=6.62 \times 10^{-34} \mathrm{Js}, c=3 \times 10^{8} \mathrm{~m} \mathrm{~Hz}$ and $k=1.38 \times 10^{-23} \mathrm{JK}^{-1}$. Equations $(\mathbb{\square})$ and $(\boldsymbol{\square})$ are such that the total energy flux in both descriptions are the same

$$
\int_{0}^{\infty} I_{\nu}(\nu, T) d \nu=\int_{0}^{\infty} I_{\lambda}(\lambda, T) d \lambda
$$

for all $T$. These integrals give rise to the well known Stefan's law for the total energy emitted by a BB [ए6]. The relation between frequency and wavelength is given by $\lambda \nu=c$. Imposing conditions for the maxima of Eqs. (四) and (『), two distinct characteristic equations are found for frequency and wavelength respectively

$$
\begin{aligned}
& \frac{x e^{x}}{e^{x}-1}-3=0, \\
& \frac{y e^{y}}{e^{y}-1}-5=0,
\end{aligned}
$$

with $x=h \nu / k T$ and $y=h c / \lambda k T$. Equations (田) and (1) are similar in form, but result in distinct roots or distinct displacement laws as a function of frequency and wavelength, respectively

$$
\begin{aligned}
\frac{h \nu}{k T} & \approx 2.8214, \\
\frac{h c}{\lambda k T} & \approx 4.9651 .
\end{aligned}
$$

As a numerical example, for $T=5500 \mathrm{~K}$ (i.e. an approximate value for the Sun's photosphere temperature), the maxima in frequency is $\nu_{\max }=325.2 \mathrm{THz}$ and in wavelength, $\lambda_{\max }=532.5 \mathrm{~nm}$. Applying the frequency wavelength relationship to the maximum in frequency, the equivalent maximum in wavelength $\left(\lambda_{\max }^{\star}\right)$ is located at $922.5 \mathrm{~nm}$. While the maximum in wavelength is in the visible part of the spectrum, the maximum in frequency is located in the near infrared region. As we will see, such maxima occur at different wavelengths depending on the used 'dispersion law'. Regardless of the particular dispersion rule chosen, all possible BB distributions still represent the same physical BB emitted spectrum.

\section{Experimental setup for measuring BB spectrum}

The relationship between emitted and measured BB spectra is governed by a transfer function that depends on a variety of physical and mathematical properties. These include, among others, transmission scattering, material dispersion and absorption rules as well as quantum responsivity of the detector as a function of frequency or wavelength. To experimentally register the BB spectrum, we need a way to separate frequency or wavelength intervals. In general, light intensity can be measured at distinct wavelengths within a small bandwidth by employing a spectrograph and a bolometer [ [20]. In the following, we present numerical estimates for the BB spectrum through different spectrograph arrangements.

\subsection{Diffraction grating spectrograph}

Fig. 四shows a schematic arrangement of this type using a diffraction grating with normal incidence. A light source $S$ is positioned in front of a slit with width $\Delta w$. The light beam is collimated by a lens $L_{1}$ with focal distance $f$ and diffracted by a grating $G$. At the detector, an image of the slit is formed. In what follows, we derive the BB spectrum distribution for a diffraction grating. The spectrum of $\mathrm{S}$ is scanned by rotating the detector arm as shown in Fig. $\mathbb{W}$ by an angle $\theta$ in relation to the undeflected direction. For reflection gratings with normal incidence, there is a straightforward and simple relationship between the wavelength and the deflection angle at first order [2]]

$$
\epsilon \sin \theta=\lambda \text {, }
$$

where $\epsilon$ is the groove spacing (lines per inch or $\mathrm{mm}$ ). We only analyse the spectrum of a single grating order. According to Eq. ( $\mathbb{\nabla})$, the grating theoretical range goes from $\lambda_{\min }=0$ to $\lambda_{\max }=\epsilon$. If $f$ is the collimator focal distance, then the wavelength resolution will be given by

$$
\delta \lambda=\epsilon \cos \theta \frac{\Delta w}{f} .
$$




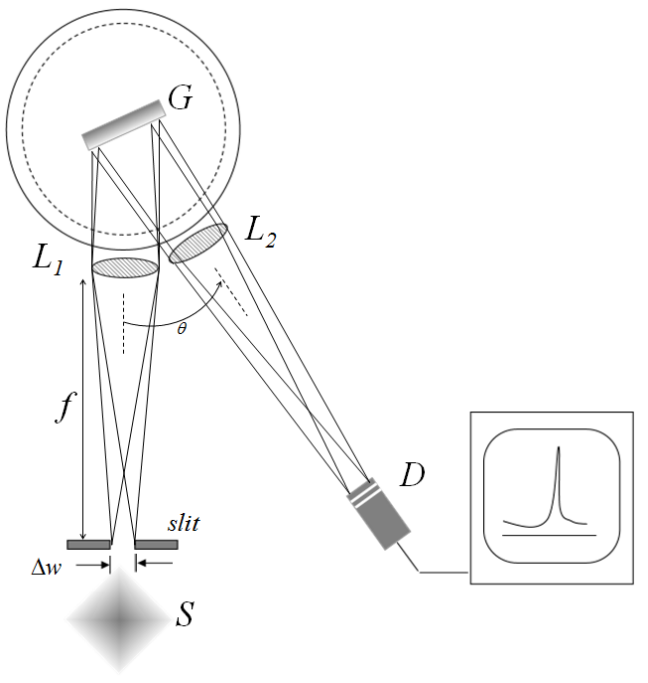

Figura 1 - Scheme of a grating spectrograph for measuring the BB spectrum.

The spectrograph measures light intensity as a function of the body emissivity and angular resolution $\delta \theta=\Delta w / f$ at the position $\theta$, or a function proportional to $I_{\theta}(\theta, T) \delta \theta$ for which

$$
I_{\lambda}(\lambda, T) d \lambda=I_{G}(\theta, T) d \theta .
$$

In reality the sensor photocurrent will be proportional to a function $F\left(I_{G} \delta \theta\right)$ that depends on many other details such as: detector response function, grating efficiency curve [[Z], sensor operational area and its distance from the grating (given by the focal distance of lens $L_{2}$ ). For instance, the detector area can be taken to be $l f_{2} \delta \theta$ where $l$ is an orthogonal dimension (sensor height) and $f_{2}$ is the focal distance of lens $L_{2}$. For simplicity, we will assume that the detector response is linear in intensity and that all other details may be represented by a proportionality parameter $\alpha<1$. Starting with Eqs. (《), (寻) and (四), the detector response (photocurrent) will be proportional to the energy flux within $d \theta$ given by

$$
I_{G}(\theta, T) d \theta=\alpha\left(\frac{2 h c^{2}}{\epsilon^{4}}\right) \frac{\cos \theta d \theta}{\sin ^{5} \theta\left(e^{h c / \epsilon k T \sin \theta}-1\right)} .
$$

The characteristic equation for the maximum of Eq. (पा) is given by

$$
\left(5 z^{2}-4 \zeta^{2}\right)-z e^{z} \frac{\left(z^{2}-\zeta^{2}\right)}{e^{z}-1}=0
$$

with

$$
z=\frac{\zeta}{\sin \theta}
$$

and $\zeta=h c / \epsilon k T$. To find a solution $z_{\max }$, first we note that Eq. (एव) can be written as

$$
A(z)=-\sum_{n=0}^{\infty}\left(\frac{\zeta}{z}\right)^{2 n+1}
$$

with

$$
A(z)=5-\frac{z e^{z}}{e^{z}-1} .
$$

In the limit of high temperatures, $\zeta / z<<1$, the root $z_{\max }$ approximates the root of Eq. (Ш)

$$
\frac{h c}{\sin \theta_{\max } k T} \approx 4.9 \text {. }
$$

In this limit, $T$ is such that $\theta_{\max }$ is between 0 and $\pi / 2$ (grazing reflectance). In general, the grating spectrograph scale will not give a simple relationship for Wien's law as in Eq. (四). Wien's peak for the spectrum given by Eq. (ㅁ) does not coincide with the wavelength peak as would be expected since the new scale introduces a nonlinear relationship for the wavelength. In other words, to get the right peak position, it is not sufficient to simply rescale the spectrograph according to Eq. (《). As a numerical example, we take a grating with 1000 grooves per millimeter so that $\lambda_{\max }=1 \mu \mathrm{m}$. The resulting $\mathrm{BB}$ spectrum is seen in Fig. (四). For $T_{1}=9000 \mathrm{~K}$ and $T_{2}=5500 \mathrm{~K}$, the solutions of Eq. ( $\square$ ) are $z_{1}=5.0782$ and $z_{2}=5.296$ corresponding to $\theta_{\max }\left(T_{1}\right)=18.35^{\circ}$ and $\theta_{\max }\left(T_{2}\right)=29.6^{\circ}$. Such values correspond to $\lambda_{\max }\left(T_{1}\right)=314.7 \mathrm{~nm}$ and $\lambda_{\max }\left(T_{2}\right)=495.6 \mathrm{~nm}$. Again, for the sun's photosphere temperature, the maximum is in the green part of the spectrum for the grating spectrograph.

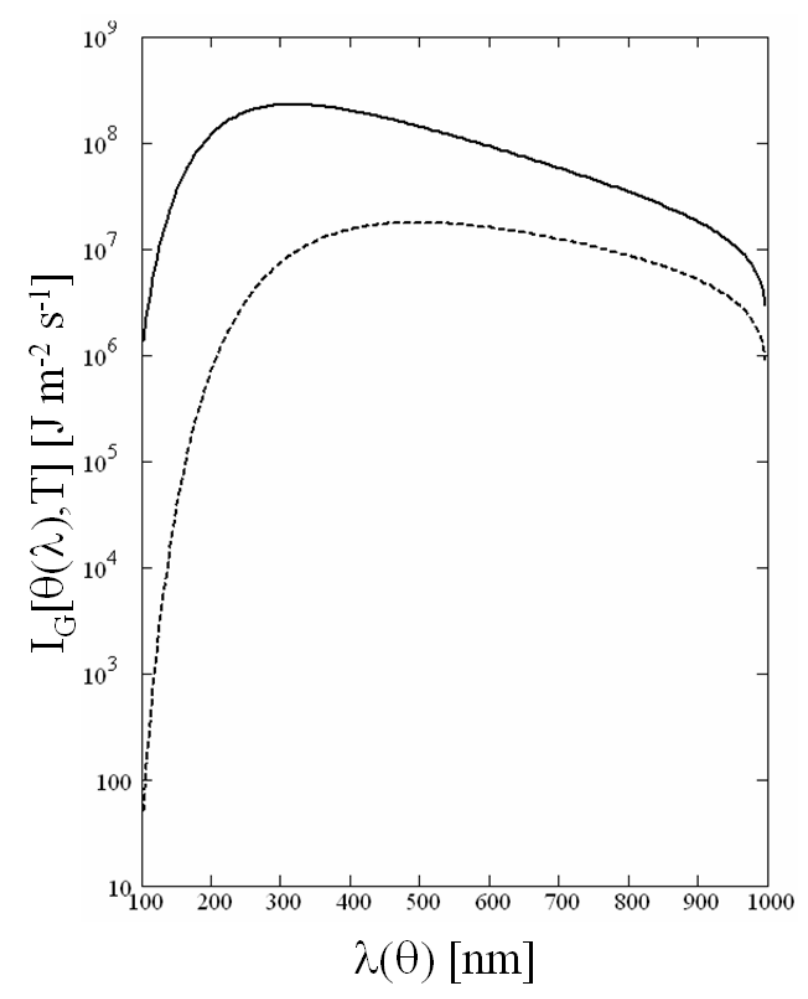

Figura 2 - BB spectrum as seen through a grating spectrograph at first order, Eq. (पII), for $T_{1}=9000 \mathrm{~K}$ (thick line) and $T_{2}=5500 \mathrm{~K}$ (dashed line). Maximum values are at $18.35^{\circ}$ and $29.6^{\circ}$, corresponding to $495.6 \mathrm{~nm}$ and $314.7 \mathrm{~nm}$, respectively. 
We can also scale the spectrograph to work in frequency. In this case, Eq. (四) will be equivalent to

$$
I_{\nu}(\nu, T) d \nu=I_{G}(\theta, T) d \theta .
$$

The frequency is given by

$$
\nu=\frac{c}{\epsilon \sin \theta}
$$

and

$$
-\frac{c d \nu}{\nu^{2}}=\epsilon \cos \theta d \theta
$$

If we multiply and divide Eq. (四) - the frequency representation - by $\nu^{2}$ we obtain Eq. (पा) again.

Since the condition for the maxium depends on $\zeta$, it is not possible to have a simple relationship as Eqs. (U) and ([) for Wien's law. As a function of $T$, solutions $\theta_{\max }$ will be given by the approximate formula

$$
\frac{h c}{\sin \theta_{\max } k T} \approx 5.24-\frac{4363}{T}+\left(\frac{5273}{T}\right)^{2}-\left(\frac{2431}{T}\right)^{3} .
$$

Eq. (खل) is of order $10^{-2}$ and is valid for a 1000 grooves/mm grating under the assumed approximations. Other gratings will have different laws with the same funcional dependence on temperature $T$.

\subsection{Prism spectrograph}

The experimental setup for a the prism spectrograph [ए2] is represented in Fig. [3. However, in this arrangement, the deviation angle is governed by refraction of light in a prism made of an optical material. Again, we assume a prism $P$ with an internal angle $A$ between faces. The light beam hits the entrance face with angle $\varphi_{1}$ (in relation to the face normal). The total deviation angle will be given by

$$
\theta=\varphi_{1}-A+\varphi_{2}
$$

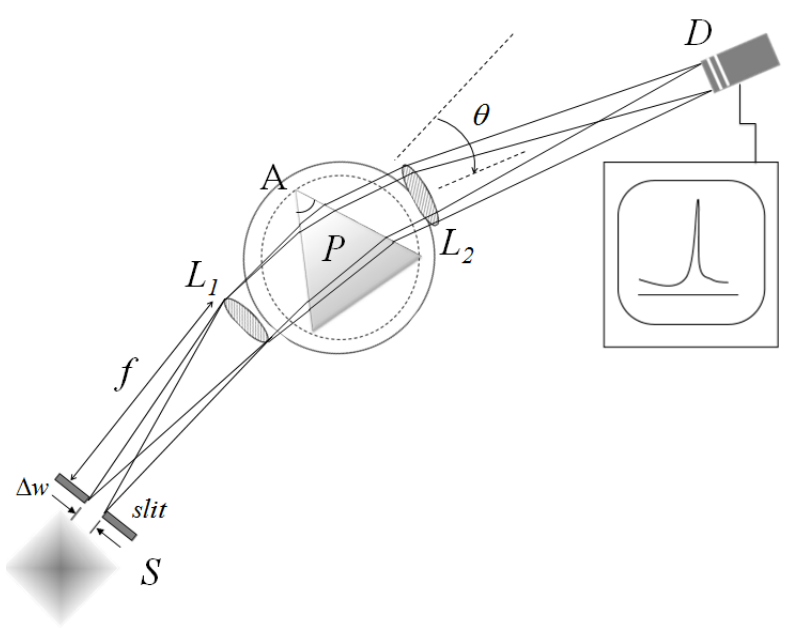

Figura 3 - Scheme of a prism spectrograph. where $\varphi_{2}$ is the refracted angle in relation to the exit face normal. Function $\varphi_{2}$ depends in fact on the wavelength according to

$$
\begin{aligned}
\varphi_{2}(\lambda) & =\arcsin \left[\sin A \sqrt{n(\lambda)^{2}-\sin ^{2} \varphi_{1}}\right. \\
& \left.-\cos A \sin \varphi_{1}\right],
\end{aligned}
$$

with $n(\lambda)$ being the prism dispersion relation. To get the material optical behavior, we use an approximate formula for Sellmeier's dispersion relation [ㅁ] ]

$$
n^{2}=a\left(1+\gamma \frac{\lambda^{2}}{\lambda^{2}-\lambda_{0}^{2}}\right)
$$

with $a=0.72, \gamma=1.91$ and $\lambda_{0}=0.085 \mu \mathrm{m}$ for Quartz. The absolute error of this aproximation in the interval $200 \mathrm{~nm}$ to $1800 \mathrm{~nm}$ is less than $0.5 \%$ as compared to the 'exact' Sellmeier's relation. For $\lambda$ in the interval $400 \mathrm{~nm}$ to $600 \mathrm{~nm}$ (corresponding to the visible region) $n(\lambda)$ goes from 1.469 to 1.457 . The relation between $\lambda$ and the total deviation angle $\theta$ is more complicated than the equivalent Eq. (四) for the grating spectrograph, but it can be summarized by the following relations

$$
\lambda(\Theta)=\lambda_{0} D(\Theta),
$$

and

$$
D(\Theta)=\sqrt{\frac{\sin ^{2} \Theta+B \sin \Theta+C}{\sin ^{2} \Theta+B \sin \Theta+C^{\prime}}},
$$

with

$$
\Theta=\theta+A-\varphi_{1},
$$

and

$$
\begin{aligned}
B & =2 \sin A \sin \varphi_{1} \\
C & =\sin ^{2} A(1-a) \\
C^{\prime} & =\sin ^{2} A[1-a(1+\gamma)]
\end{aligned}
$$

We here write the $\mathrm{BB}$ spectrum distribution in the prism reference as determined by the dispersion relation Eq. (एव). As usual, we start with the wavelength form Eq. (四) by noting that $d \theta=d \Theta$, we have

$$
I_{P}(\Theta, T)=\left(\frac{2 h c^{2}}{\lambda_{0}^{4} D(\Theta)^{5}}\right) \frac{1}{e^{h c / \lambda_{0} D(\Theta) k T}-1}\left|\frac{d D}{d \Theta}\right| d \Theta,
$$

with

$$
\frac{d D}{d \Theta}=\frac{\left(C^{\prime}-C\right) \cos \Theta(2 \sin \Theta+B)}{2 D(\Theta)\left(\sin ^{2} \Theta+B \sin \Theta+C^{\prime}\right)^{2}} .
$$

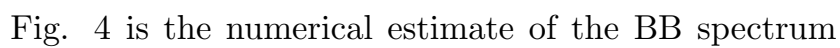
distribution as given by Eq. (एש) for a prism with $A=60^{\circ}$ and entrance angle $\varphi_{1}=45^{\circ}$. To obtain the new peak position at a certain temperature $\mathrm{T}$, one must again impose $d I_{P} / d \Theta=0$ to Eq. (酋). To avoid unnecessary complications, we provide numerical values only. As shown in Fig. 13, a BB peak for 
$T=5500 \mathrm{~K}$ is at $\theta_{\max }=21.27^{\circ}$. Using the prism dispersion relation, Eq. (四), the corresponding wavelength is $\lambda_{\max }=1671.5 \mathrm{~nm}$ which is again in the infrared part of the spectrum.

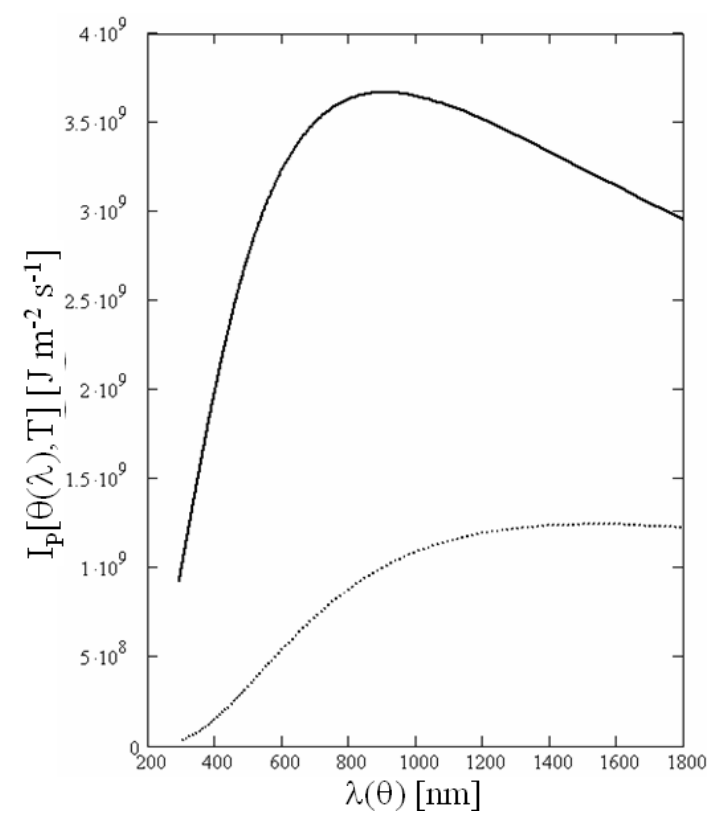

Figura 4 - BB spectrum curves of a Quartz prism spectrograph,

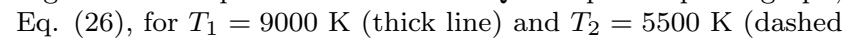
line). Maximum values are at $21.42^{\circ}$ and $21.27^{\circ}$, corresponding to $\lambda_{\max }\left(T_{1}\right)=1025.7 \mathrm{~nm}$ and $\lambda_{\max }\left(T_{2}\right)=1671.5 \mathrm{~nm}$, respectively.

\section{Generalization}

The results obtained so far can be generalized. Suppose two functions $f_{1}(x)$ and $f_{2}(y)$ are given such that

$$
f_{1}(x) d x=f_{2}(y) d y
$$

together with the 'dispersion relation'

$$
y=g(x)
$$

Then

$$
f_{1}(x)=f_{2}(g(x)) g^{\prime}(x),
$$

where $g^{\prime}(x)=d y / d x$. Imposing the condition for the maximum at $\bar{x}$

$$
\left.f_{1}^{\prime}(x)\right|_{x=\bar{x}}=0
$$

we find

$$
\left.\frac{d f_{2}}{d y}\right|_{\bar{y}=g(\bar{x})}=-\frac{f_{2}(g(\bar{x})) g^{\prime \prime}(\bar{x})}{g^{\prime}(\bar{x})^{2}},
$$

that is clearly not zero, unless $f_{2}(g(\bar{x}))=0$ or $g^{\prime \prime}(\bar{x})=0$ (that is, this is not the case for the relationship between frequency and wavelength). Suppose that we further make a transformation for a new variable $z$ so that

$$
x=h(z) .
$$

We call $z$ the 'experimental scale' (as in the case of frequency or wavelength scales). The following relations are valid

$$
\begin{aligned}
& f_{1}(x) d x=f_{3}(z) d z, \\
& f_{2}(y) d y=f_{3}(z) d z .
\end{aligned}
$$

Then, in terms of $f_{1}(x)$

$$
\frac{d f_{3}}{d z}=f_{1}^{\prime}\left(\frac{d h}{d z}\right)^{2}+f_{1} \frac{d^{2} h}{d z^{2}} .
$$

In terms of $f_{2}(y)$

$$
\frac{d f_{3}}{d z}=\left(\frac{d f_{2}}{d y} g^{\prime 2}+f_{2} g^{\prime \prime}\right)\left(\frac{d h}{d z}\right)^{2}+f_{2} g^{\prime} \frac{d^{2} h}{d z^{2}} .
$$

Given Eq. (四), both ways of calculating the maximum of $f_{3}(z)$, Eqs. (\$6) and (\$7) lead to the same value. Eqs. (\$6) and (B]) also show that the condition $d f_{3}(\bar{z}) / d z=0$ does not led to $d f_{2}(\bar{y}) / d y=0$ and $f_{1}^{\prime}(\bar{x})=0$. The derivatives at the extremum $\bar{y}=g(\bar{x})$ can be found by imposing $d f_{3} / d z=0$ in Eqs. (피) and (B]) and extracting both $f_{1}^{\prime}(\bar{x})$ and $d f_{2}(\bar{y}) / d y$

$$
\begin{gathered}
\left.\frac{d f_{1}}{d x}\right|_{\bar{x}}=-f_{1}(\bar{x})\left(\frac{d^{2} h}{d z^{2}}\right)\left(\frac{d h}{d z}\right)^{-2}, \\
\left.\left.\frac{d f_{2}}{d y}\right|_{\bar{y}}=\frac{1}{g^{\prime}(\bar{x})^{2}}\left[f_{2}(\bar{y}) \frac{d^{2} h}{d z^{2}}\left(\frac{d h}{d z}\right)^{2}+f_{2}(\bar{y}) g^{\prime \prime}(\bar{x})\right)\right] .
\end{gathered}
$$

A somewhat more prosaic example is the search for the maximum position through a new 'gauge', say, $y=x^{2}$, knowing that

$$
f_{1}(x)=\frac{1}{\sigma \sqrt{2 \pi}} e^{-(x-\mu)^{2} / 2 \sigma^{2}} .
$$

To be more specific, suppose that a distribution of bar lengths was found to be in accordance to the normal rule of Eq. ( 38$)$ with $\bar{x}=\mu$. At this point, the maximum value of Eq. (Bष) is reached, that is $d f_{1}(\bar{x}) / d x=0$. In the new 'squared' scale, the distribution will be

$$
f_{2}(y)=\frac{1}{2 \sigma \sqrt{2 y \pi}} e^{-(\sqrt{y}-\mu)^{2} / 2 \sigma^{2}},
$$

which has as characteristic equation $d f_{2}(y) / d y=0$

$$
y-\mu \sqrt{y}+\sigma^{2}=0 .
$$

Solutions are

$$
\bar{y}_{ \pm}=\frac{\mu^{2}}{4}\left(1 \pm \sqrt{1-4 \frac{\sigma^{2}}{\mu^{2}}}\right)^{2} .
$$

The distribution given by Eq. (Bप) diverges as $y \rightarrow 0$. If, for example, $\mu=10$ and $\sigma=2$, then $\bar{y}_{+}=91.82$ corresponds to the peak at the 'intuitive' maximum position $\bar{y}=100$. Such maximum in $y$ depends on the variance $\sigma^{2}$, and only when $\sigma \rightarrow 0, \bar{y} \rightarrow \bar{x}^{2}$. If we disregard the region $y<<1$, for $\mu<2 \sigma$, there is no real extrema for the distribuition of Eq. (उप). At $\mu=2 \sigma$, 
there is only one extremum at $\bar{y}=\mu^{2} / 4$. Such simple example shows that when the scale changes acording to some nonlinear function, the extrema chage position and are not described by the simple nonlinear relationship between scales.

Now, if we use another scale $(z)$ with $x=10^{z}$, the resulting distribution $f_{3}(z)$ will be

$$
f_{3}(z)=\left(\frac{\ln (10)}{\sigma \sqrt{2 \pi}}\right) 10^{z} e^{-\left(10^{z}-\mu\right)^{2} / 2 \sigma^{2}} .
$$

The characteristic equation, $d f_{3}(z) / d z=0$, in this case is

$$
10^{2 z}-\mu 10^{z}-\sigma^{2}=0 .
$$

Again, a real solution can be written as

$$
\bar{z}_{+}=\log \left(\frac{\sqrt{\mu^{2}+4 \sigma^{2}}-\mu}{2}\right) .
$$

For the numerical example given above $(\mu=10$ and $\sigma=2), \bar{z}=1.016$ which neither coincides with $\bar{z}(\bar{x}=10)=1$, as expected by the $x=10^{z}$ law, or $\bar{z}(\bar{y}=91.82)=0.981$ as expected by the $z=\log (\sqrt{z})$ relationship. Fig. 1 represents plots of functions $f_{1}(x)$, $f_{2}(y)$ and $f_{3}(z)$ for two distinct values of $\sigma$. For $f_{2}(y)$, the maximum position shifts to the left as $\sigma$ increases, while the opposite happens to $f_{3}(z)$.
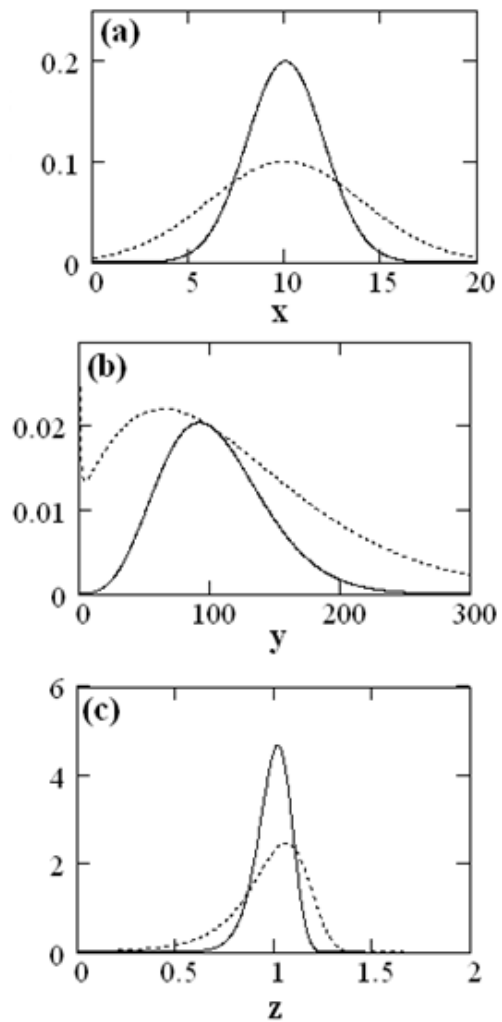

Figura 5 - Plot of $f_{1}(x)$ for $\mu=10, \sigma=2$ (thick line) and $\sigma=4$ (dashed line). For the normal distribution, the maximum position does not depend on variance. (b) Plot of $f_{2}(y)$. The maxium shifts to the left as $\sigma$ increases. (c) Plot of $f_{3}(z)$. The opposite of (b) happens.
In general, the maxima will hardly coincide, although the transformation to a third 'experimental' function shows only one maximum (if there is only one extremum). In the same sense, the spectrum of Eqs. (『) and $(\mathbb{U})$ can only be measured if an experimental device with linear scales for $\lambda$ or $\nu$ becomes available.

\section{Conclusion}

This paper addresses and answers the question: "where is the Wien's peak?' [ए]]. The emitted power density maxima are derived from Planck's distribution law described either in frequency or wavelength intervals. We showed that such emitted power of a BB body at absolute temperature $T$ is unique and does not coincide with the measured spectra (in fact we present theoretical estimates of measurable values). Estimated spectral shifts are caused by nonlinear frequency or wavelength 'gauge' relations to the experimentally accessible parameter (an intensity within an interval of such parameter). In general, there is no simple relation between the temperature and the maximum position (Wien's law) in the spectrograph scale given by this parameter. For prism spectrographs, the highly non linear dispersion relation complicates the task of obtaining a simple relationship between the maximum position and the temperature. We have found that the maximum position (in fact, the spectrum distribution) depends on the way that light interact with the dispersing element (i.e., prism or grating). The more linear the dispersion relation is, the closer we get to the Planck's distribution, Eq. (回).

All these conclusions are valid under ideal assumptions, which means to assume achromatic response for the lenses and sensing elements. If such assumptions are relaxed, then Eq. (प) will not be valid. The spectrum will be modulated by the transfer function of the combined spectrograph elements and the maximum will be found againg by imposing the condition $d F(\theta) / d \theta=0$ where $F(\theta)$ is the output spectrum.

In spite of the nonlinear dependency of the BB radiation spectrum function on $\nu$ or $\lambda$, the shift observed for the peak can be easily demonstrated with other distribution fuctions under nonlinear scales.

\section{References}

[1] M. Planck, Annalen der Physik. 4, 553 (1901).

[2] The work deservedly rendered him the Nobel prize of physics in 1918, see http://nobelprize.org/

[3] O. Lummer and E. Prigsheim, Verh. d. D. Phys. Gesell. 1, 215 (1899).

[4] O. Lummer and E. Prigsheim, Verh. d. D. Phys. Gesell. 2, 163 (1900).

[5] W.H. Cropper, The Quantum Physicists (Oxford, New York, 1970). 
[6] A. Sommerfeld, Thermodynamics and Statistical Mechanics - Lectures on Theoretical Physics, v. 5 (Ney York Academid Press, New York, 1956), Eq. (20a) on p. 144

[7] R.K. Pathria, Statistical Mechanics (ButterworthHeinemann, Oxford, 1996), 2nd ed., Eq. (8) on p. 170.

[8] C. Kittel, Elementary Statisticlal Physics (John Wiley and Sons, New York, 1958), Eq. (22.7) on p. 104.

[9] S.R.A. Salinas, Introduction to Statistical Physics (Springer, New York, 1997), Eq. (10.91) on p. 206.

[10] B.M. Smirnov, Principles of Statistical Physics - Distributions, Structures, Phenomena, Kinetics of Atomic Systems (Wiley VCH, Weinheim, 2006), Eq. (3.3) on p. 28.

[11] L.D. Landau and E.M. Lifshitz, Course of Theoretical Physics - Statistical Physics (Pergamon Press, Oxford, 1980), v. 5, Part I, 3rd ed., Eq. (64.9) on p. 184.

[12] F. Reif, Fundamentals of Statistical and Thermal Physics (McGraw-Hill Book Company, New York, 1963), Eq. (9.13.10) and (9.13.13) on p. 375 and p. 376, respectively.

[13] J. Jeans, The Dynamical Theory of Gases (Dover Publication, New York, 1925), 4th ed., Eq. (933) on p. 372 and Eq. (930) on p. 371.
[14] P.T. Landsberg, Thermodynamics and Statistical Mechanics (Dover Publications, New York, 1978), 2nd ed., p. 214, Section 13.3 .

[15] C.E. Mendenhall and F.A. Saunders. Astrophys. Journal, 13, 25 (1901).

[16] I. Kaplan, Nuclear Physics (Addison-Wesley Pub., New York, 1964), 2nd ed.

[17] M.A. Heald, Am. J. Phys. 71, 1322 (2003).

[18] J.M. Overduin,Am. J. Phys.71, 216 (2003).

[19] D.J. Fixsen, E.S. Cheng, J.M. Gales, J.C. Mather, R.A. Shafer and E.L. Wright, Astrophysical Journal 473, 576 (1996).

[20] R.C. Jones, J. Opt. Soc. Am. 43, 1 (1953).

[21] W. Sellmeir, Ann. d. Phy. u. Ch. 219, 271 (1871).

[22] J. James, Spectrograph Design Fundamentals (Cambridge Univ. Press, Cambridge, 2007).

[23] A.H. Wilson, Thermodynamics and Statistical Mechanics (Cambridge University Press, Cambridge, 1957), Eq. (6.52.3) on p. 184.

[24] C. Palmer. Diffraction grating Handbook (Thermo RGL, Rochester, 2002), 5th ed.

[25] J. Ma, J. Yang and J. Nie. Lat. Am. J. Phys. Educ. 3, 566 (2009). 